\title{
PERSPECTIVES POUR DES SÉMIOTIQUES DE LA PHOTOGRAPHIE ${ }^{1}$
}

\author{
Maria Giulia Dondero ${ }^{2}$
}

Mon étude voudrait faire le point sur les pratiques descriptives que la discipline sémiotique a conduites sur la photographie, autant sur la photographie en tant que texte -je fais référence surtout à la sémiotique greimassienne, en particulier à l'œuvre de Jean-Marie Floch Les formes de l'empreinte ${ }^{3}$, , que sur la photographie en tant que medium -je fais référence notamment aux théories de tradition peircienne 4 .

Premièrement, je me demanderai comment décrire un texte photographique et en même temps comment rendre compte sémiotiquement de ses pratiques productives et réceptives - lesquelles rendent descriptible le texte lui-même.

Deuxièmement, j'essaierai de montrer comment - à travers une brève investigation autour des théories de Greimas et de Floch d'un côté, et de Peirce de l'autre - on pourrait esquisser une typologie de

1 Je remercie vivement Nathalie Roelens et François Provenzano pour la correction très attentive de cet article.

2 Post-doctorante (FNRS/Université de Liège).

3 J.-M. FLOCH, Les formes de l'empreinte, Périgueux, Pierre Fanlac, 1986.

4 Ph. DuBOIS, L'acte photographique, Bruxelles, Labor, n. e. Paris, Nathan, 1990.

Recherches en communication, $\mathrm{n}^{\circ} 27$ (2007). 
différents genres photographiques et de leurs statuts et montrer ainsi comment les niveaux des genres et des statuts, avec celui du texte, peuvent construire des niveaux fondamentaux pour une stratification analytique du champ de la photo.

\section{La sémiotique de la photographie entre spécificité du medium et textualité}

Le regard sur la photographie n'est pas seulement l'apanage des théories sémiotiques greimassiennes et peirciennes. Le domaine de la photographie a été interrogé par différentes disciplines : sociologiques, historiques, historico-philosophiques, historico-artistiques, etc. Le domaine de la photographie pourrait être considéré comme un lieu de débat et de confrontation entre différents types de description, méthodologies, théories, épistémologies.

Les théories sociologiques, esthétiques, ou les approches historico-culturelles, lorsqu'elles se sont référées aux questions sémiotiques posées par la photographie, l'ont fait dans les vieux termes de la définition d'une spécificité, en partant du travail de Roland Barthes ${ }^{1}$. Les disciples de la sémiotique peircienne, tels Philippe Dubois ou Rosalind Krauss $^{2}$, ont également mis au centre de leur recherche l'étude de la spécificité médiale de la photographie. Cette approche de la spécificité du langage et du médium est, paradoxalement, une approche généralisante, qui ne porte aucune attention aux textualités et qui va en direction opposée par rapport aux programmes d'une sémiotique du texte de type caractérisante, analytique, de dérivation structuraliste et greimassienne.

La théorie greimassienne, rejetant une distinction fondée sur la spécificité des modes de production de textes, donc une distinction médiatique, a pu affiner les instruments d'analyse d'un texte à partir du parcours génératif du contenu ${ }^{3}$. La théorie greimassienne a construit une théorie de l'analyse qui vise à tracer, comme le dit Pierluigi Basso, « une trajectoire définie afin d'activer des processus de sémantisation

1 R. BARTHES, La chambre claire. Note sur la photographie, Paris, Gallimard, 1980.

2 R. KRAUSS, Le photographique. Pour une théorie des écarts, Paris, Ed. Macula, 1990.

3 A.-J. GREIMAS, Du sens II. Essais sémiotiques, Paris, Seuil, 1983. 
le plus objectifs possible pour diriger le récepteur [...]. Cette trajectoire de sens montrée par l'analyse sert à construire et à stabiliser un cadre descriptif négociable comme fond intersubjectif tourné vers la commensurabilité de nos différentes interprétations $»^{1}$.

Dans le projet greimassien, l'objectif des analyses textuelles est de parvenir à une description des parcours sémantiques que chaque texte active et de construire par conséquent une validité intersubjectivement négociable des descriptions. Cette approche a servi à s'éloigner de l'impressionnisme interprétatif, du psychologisme, mais elle a laissé de côté deux axes de recherche importants : l'étude des techniques productives et des pratiques interprétatives culturellement incarnées.

S'il a été possible, pour la tradition greimassienne, d'étudier la textualité, qui objectivise les pratiques, il a paru plus difficile d'analyser les pratiques elles-mêmes, qui relèvent de l'idiosyncrasie des techniques et des situations d'énonciation et de réception.

\section{La photographie en tant que texte : Greimas et Floch}

J'aborderai à présent la caractérisation de la théorie greimassienne par rapport à la théorie peircienne sur la photographie, même si en réalité, dans le cas de Greimas et de Floch, il ne s'agit pas d'une théorie portant explicitement sur la photographie, mais plutôt d'une théorie du discours - qui peut être convoquée pour étudier des textes photographiques.

La tradition greimassienne s'est désintéressée de la prise en compte de la production du texte, question qui est au contraire au centre de la sémiotique d'inspiration peircienne. La théorie du discours de Floch et Greimas étudie la photographie comme tout autre type de texte, à partir du plan du contenu des textes, et exclut de l'analyse la question de la formation du plan de l'expression. En effet, dans Formes de l'empreinte Floch propose de mener :

une recherche sur une typologie des discours aussi bien nonverbaux que verbaux qui, de fait, intègrerait $l$ ' « histoire intérieure des formes » de la photographie, et plus généralement de l'image, à celle de tous les langages, de toutes les sémiotiques. Un tel projet d'intégration est d'ailleurs tout à fait typique d'une

1 P. BASSO, Confini del cinema. Strategie estetiche e ricerca semiotica, Torino, Lindau, 2003 (Traduction de l'auteur). 
sémiotique structurale et confirme une fois de plus l'antinomie entre cette dernière et une sémiologie des signes et de leur spécificité respective ${ }^{1}$.

La production photographique, à savoir la genèse à empreinte, est laissée aux marges de l'analyse et elle n'est récupérée que comme énonciation énoncée, comme pli métalinguistique. Le refus de considérer la technologie et les pratiques d'instanciation est fortement lié à la négation d'une corporéité énonciative dont, en revanche, il faut s'occuper, même dans le cas de l'acte photographique, où la production renvoie à un actant dual, à savoir à un hybride humain et non-humain².

Dans l'essai de Floch, les analyses sémiotiques des photographies mettent en évidence le rôle crucial de la lecture plastique qui permet à Floch de démontrer comment l'image produit un effet de sens qui est lié aux formes de l'empreinte et non pas à la genèse de l'empreinte qui l'a produite. A ce propos Floch affirme que : "Si d'un point de vue technique, l'image photographique peut être considérée comme une empreinte, ce sont les formes de l'empreinte qui rendent possible le fonctionnement de l'image en tant qu'objet de sens » (p. 170) ${ }^{3}$.

Cependant, même s'il fait de l'analyse plastique des textes ${ }^{4}$, Floch part d'une théorie du discours qui ne prend pas en compte la substance du plan de l'expression dans la sémantisation du texte. Par exemple, dans l'analyse du $N u n^{\circ} 53$ de Brandt, Floch rapproche l'esthétique de la découpe de Brandt et celle de Matisse (en particulier dans ses gouaches découpées des années 50), affirmant que l'effet de sens des formes photographiques de Brandt construites sur des éléments plats et des lignes-contours (à la manière des décorateurs égyptiens) équivaut à l'effet de sens des configurations des gouaches de Matisse. Pour affirmer, avec raison, que le procédé photographique n'implique aucune forme plastique particulière, il soutient que l'esthétique de la

1 J.-M. FLOCH, Les formes de l'empreinte, op. cit., p. 106-107 (c'est moi qui souligne).

2 Je me permets de renvoyer à ce propos à M. G. DONDERO, « Les pratiques photographiques du touriste entre construction d'identités et documentation ", Communication et Langage, 151, 2007, pp. 21-38.

3 J.-M. FLOCH, « The arms of the moon itself. Plastic Description of the Photograph Nude ${ }^{\circ} 53$ by Bill Brandt », Semiotics, n. 15-16, 2001, pp. 168-186 (c'est moi qui traduis et souligne, MGD).

4 Sur l'analyse plastique, voir A. J. GREIMAS, « Sémiotique figurative et sémiotique plastique », Actes sémiotiques - Documents, n. 60, 1984. 
découpe est une esthétique qui fonctionne de la même manière dans les différentes substances expressives, comme la peinture, la photographie, mais aussi le cinéma, la littérature, etc. - et cela est moins juste à mon sens ${ }^{1}$.

Il est clair que la constitution du plan de l'expression, dans le premier cas liée à l'empreinte de la lumière sur un support sensible et dans le second cas à la technique à gouache, n'a aucune pertinence sémiotique pour Floch. Il est vrai qu'autant chez Matisse que chez Brandt, le corps en tant que figurativité disparait ; seule reste l'empreinte eidétique, l'abstraction de la corporéité, mais la relation fondamentale entre les techniques de l'apport et le traitement différencié du support dans le cas de la photo et dans le cas de la gouache n'est en aucun cas prise en considération.

Floch met en parallèle et identifie des formes sémiotiques communes au delà de la substance expressive employée ${ }^{2}$. Cette démarche est motivée par le fait que Floch considère exclusivement la pertinence 《 visuelle » des images - et met entre parenthèses le caractère corporel polysensoriel, tant du substrat matériel d'inscription que du geste d'énonciation ${ }^{3}$. Je suis convaincue, au contraire, qu'il faudrait supposer certaines « asymétries » des formes sémiotiques, à partir des différentes substances expressives où ces formes elles-mêmes s'incarnent. Mais ce ne sont pas seulement les différentes pratiques de production - c'est-àdire le fait que l'image photographique et que l'image picturale mettent en scène des textures différentes - qui sont en jeu ici, mais c'est aussi le fait que ces deux gestes de production supportent des habitudes perceptives et interprétatives différentes.

Nous devons donc nous demander : la théorisation de Floch contenue dans Formes de l'empreinte, qui nie la spécificité de la photographie, peut-elle être vraiment valable pour toutes les autres textualités, picturales, cinématographiques, graphiques, etc. ?

1 Comme le dit très clairement Floch : «Selon nous, en effet, le meilleur service à rendre aujourd' hui à la photographie, c'est l'intégrer au monde des images en général et insister sur le fait qu'elle est traversée, si l'on peut dire, par des nombreuses esthétiques ou formes sémiotiques qui se prolongent aussi bien dans la peinture, le dessin ou le cinéma » (Les formes de l'empreinte, op. cit., p. 115).

2 Cette conception de Floch reprend H. WÖLFLIN, Principes fondamentaux de l'histoire de l'art. Le problème de l'évolution du style dans l'Art Moderne, Paris, Gérard Monfort, 1992.

3 Voir à ce sujet J. FONTANILLE, Soma et séma. Figures du corps, Paris, Maisonneuve et Larose, 2004. 
Étant donné que, chez Floch, l'instance énonciative est désincarnée, il devient impossible de rendre compte de la différence sémiotique entre la corporéité machinique impliquée dans la prise d'une photo et la sensori-motricité impliquée dans la genèse d'une aquarelle. Il devient par conséquent difficile de rendre compte de la relation entre la textualité et les pratiques de production, qui permettrait en revanche d'analyser la photographie en tant qu'objet culturel situé dans une tradition productive (et interprétative).

Contrairement à Floch, et selon une nouvelle approche sémiotique, on pourrait considérer que les formes textuelles ne sont plus des configurations déposées et désincarnées, mais bien des formes imprégnées des «manières du faire » et d'une activité corporelle. On arriverait ainsi à considérer les articulations du contenu des images à partir des variations de la substance de l'expression et de sa prise de forme. Il faudrait prendre en compte les différents gestes de production sans pour autant tomber dans l'ontologisation de la genèse de l'empreinte.

\section{La photographie comme médium. Les disciples de Peirce}

La tradition des études peirciennes, contrairement à la tradition des études greimassiennes, s'est occupée principalement de la production du signe, étudiant ainsi la photographie à partir de son lien avec le référent. Comme le rappelle Dubois (1983), cette relation a été pensé en trois temps : d'abord en termes de ressemblance iconique, ensuite ré-attribuée, sous l'impulsion de la polémique anti-iconique, à des conventions symboliques, et enfin, conçue en termes de connexion indexicale. Dans ces passages, le rappel à la plus connue des tripartitions peirciennes (icône, index, symbole) est presque toujours explicite.

Du côté peircien, la photographie est donc considérée en tant qu'index et empreinte d'un fragment de monde, « connexion et division du signe avec son référent », comme l'affirme le même Dubois dans son ouvrage. Cette théorie de l'indexicalité se révèle cependant être seulement un «passe-partout » qui généralise et ontologise le discours sur la photographie, et ne rend compte ni des formes de l'empreinte - c'està-dire des configurations textuelles -, ni des pratiques de réception. La théorie de l'index photographique sert à Dubois et aux autres peirciens à « expliquer » toutes les images photographiques et, par conséquent, aucune, parce que cette théorie n'est pas « mise à l'épreuve » par les différentes textualités. C'est une théorie qui ne se confronte pas avec la variété des occurrences concrètes et avec leurs statuts : c'est une théorie 
construite non pas sur des textes attestés, des textes en situation, mais au contraire sur des textes possibles (prévisibles) qui sont, comme l'affirme Rastier, «privés de situation - et donc dépourvus de sens » ${ }^{1}$.

En effet, la tripartition peircienne a toujours été associée à des propriétés ontologiques du signe photographique. Il aurait été bien plus heuristique de considérer les termes de cette tripartition comme trois manières de constituer les pertinences d'un même objet, comme l'affirme Pierluigi Basso Fossali dans un de ses articles sur Peirce ${ }^{2}$ : le signe peut être reconduit, dans le cas de la priméité, à une configuration diagrammatique qui exemplifie des propriétés, dans le cas de la secondéité, au résultat d'un processus constituant, et enfin, dans le cas de la tiercéité, à un membre d'une mémoire culturelle. Ces pertinences assumées localement concernent des axes d'assomption du signe et non des propriétés ontologiques.

D'un côté nous avons donc la sémiotique greimassienne qui postule à l'origine du sens une absence et une instance énonciative vide, et qui étudie les textes d'un point de vue de survol, désincarné ; du coté peircien il existe une opération d'ontologisation de la genèse ; dans tous les cas il s'agit d'opérations d'abstraction du sens.

Ce bref parcours à travers les différentes théories sémiotiques nous montre que ni la tradition d'étude de Greimas ni la tradition théorique de Peirce n'arrivent, pour des raisons différentes, à rendre compte des niveaux intérmédiaires entre texte et genèse du texte-même qui leur permettraient d'analyser enfin le texte photographique et ses relations intertextuelles, intermédiales et socio-culturelles.

Si la sémiotique de Floch analyse des textualités photographiques sans tout à fait s'interroger sur les différents genres et statuts de l'image, ou sur les différentes assomptions statutaires d'une même image, c'est parce qu'elle ne donne pas d'ancrage situationnel au texte : elle le dépourvoit de son rattachement à son histoire de production et à ses encadrements sociaux - ancrage nécessaire pour pouvoir rendre compte des pratiques interprétatives de la photo.

De son coté, la sémiotique d'inspiration peircienne a consacré son attention à la génétique de la production, à la spécificité médiale, en

1 F. RASTIER, Arts et sciences du texte, Paris, PUF, 2001, p. 98.

2 P. BASSO FOSSALI, « Peirce e la fotografia : abusi interpretativi e ritardi semiotici » dans P. BASSO FOSSALI \& M. G. DONDERO, Semiotica della fotografia. Investigazioni teoriche e pratiche di analisi, Rimini, Guaraldi, 2006, pp. 113-214. 
excluant de son horizon la particularité des textes et la description des genres et des différentes pratiques en réception.

Je me propose maintenant de partir de la prise en compte de la génétique des textes photographiques (sans l'ontologiser !) pour revenir enfin sur les genres et les statuts des textes.

\section{Art et reproductibilité photographique}

Tout le monde a toujours opposé l'image artistique à l'image documentaire. On a eu des théoriciens qui ont supporté cette opposition en partant d'une distinction de nature ontologique, c'est-à-dire à partir de la considération que les photos artistiques sont artistiques en raison de leur configuration visuelle (virtuosité, expérimentation formelle, etc.).

D'autres théoriciens sont partis au contraire d'une distinction qui prend en compte les pratiques de production et d'utilisation des images, comme, par exemple, la sacralisation de l'original unique dans le cas de la photo artistique. Cette sacralisation n'est pas pertinente dans le cas de la photographie documentaire, consacrée à la mise en scène d'événements d'actualité. Ici le tirage original n'a aucune importance et aucune pertinence en termes de signification : des photos de presse on peut faire autant de tirages qu'on veut et on peut même les cadrer différemment en en coupant des parties. Au contraire, dans le cas de l'image artistique, considérée comme autographique selon les termes de Nelson Goodman ${ }^{1}$, chaque zone, chaque détail et même les bords de l'image sont censés syntaxiquement et sémantiquement denses. Mais, pour mieux comprendre cette affirmation, arrêtons-nous un moment sur les pratiques productives et interprétatives de la photo artistique.

La photographie a été légitimée comme art très tard et cela pour différentes raisons. Ce qui, entre autres, a empêché pour longtemps la photographie de rentrer dans le domaine de l'art est sa reproductibilité. La photographie a été pensée et théorisée au début comme image qui ne possède pas d'original, si on entend par original un texte unique où sont inscrites les traces de l'acte sensori-moteur de production - comme il arrive dans le cas des traces de la main du peintre et de son geste non répétable sur le tableau. Selon cette conception (contenue en La petite

1 N. GoOdman, Les langages de l'art. Une approche de la théorie des symboles, Paris, Hachette, 2005 (1968). 
histoire de la Photographie de Benjamin $)^{1}$, il n'y a que le négatif ou le daguerréotype qui puissent devenir des œuvres d'art, parce qu'ils sont liés directement à l'acte productif : seul à l'original de l'œuvre on peut attribuer un surplus de sens, c'est-à-dire une portée sémantique liée à la trace sensible de son instanciation. L'aura dont parle Benjamin c'est précisément ce qui met en relation le présent de la réception avec le passé de la production - en effet l'aura est définie comme « une trame singulière d'espace et de temps : unique apparition d'un lointain, si proche soit-il » (p. 70). Tel surplus de sens qu'engendre l'œuvre originale conservée dans un musée est donné par la stricte relation entre le parcours productif de l'œuvre et le parcours de l'observation en acte, comme le dit Pierluigi Basso dans Il dominio dell'arte ${ }^{2}$.

Selon la théorie de Benjamin, on devrait conclure que, si à partir du négatif on peut produire une série de tirages différents, la question $d u$ tirage authentique n'a pas de sens. Pour le théoricien allemand la sacralité auratique de l'original s'épuise, se consomme et perd son intensité à cause de l'extension quantitative des différents tirages. De plus, la photographie, par rapport à la peinture, est considérée à l'instar d'un art mécanique, qui n'est pas tracé par une sensori-motricité anthropomorphe ${ }^{3}$.

Si nous prenons en considération la distinction formulée par le philosophe Nelson Goodman entre les arts autographiques, dont l'authentification est liée à l'histoire productive, et les arts « allographiques », authentifiables à travers « l'identité orthographique » (sameness of spelling), l'image photographique pourrait être qualifiée d'œuvre autographique à objet multiple. Pour nous, au contraire de Benjamin, la photographie est autographique, comme l'est la peinture, parce que les formes de l'empreinte photographique renvoient à l'histoire de sa production, donc à un événement corporel unique ${ }^{4}$. On doit toutefois

1 W. Benjamin, L'œuvre d'art à l'époque de sa reproductibilité technique, Paris, Allia, 2003 (1936).

2 P. BASSO, Il dominio dell'arte. Semiotica e teorie estetiche, Roma, Meltemi, 2007.

3 Cette théorie ne rend pas compte du fait que l'appareil photographique est toujours en relation avec le corps du photographe qui prend position dans le monde environnant : l'image photographique n'est pas le produit d'un faire mécanique, mais d'un faire mixte, anthropomorphe et machinique.

4 Sur la question de l'original photographique, je me permets de renvoyer le lecteur à M. G. DONDERO, Fotografare il sacro. Indagini semiotiche, Roma, Meltemi, 2007 et à M. G. DONDERO, «Quand l'écriture devient texture de l'image », Visible n 2, pp. 11-32. 
préciser que les différents tirages que l'on peut obtenir à partir d'un seul négatif (autographique) sont multiples et authentiques (c'est pour cela qu'on appelle les photos des « œuvres autographiques à objet multiple $)^{1}$. Cela signifie que le photographe doit choisir une technique et un calibre du tirage qui puissent individualiser l'original photographique. Grâce aux choix de tirage du photographe, les tirages photographiques ne seraient pas de simples occurrences du négatif, voire des exécutions d'une partition comme c'est le cas de la musique qui est l'art allographique par excellence, mais au contraire ils accéderaient au statut d'œuvres autonomes et non reproductibles, à savoir autographiques $^{2}$.

\section{Entre statuts documentaire et artistique, les "genres mixtes"}

A partir de la distinction entre statut documentaire et artistique, beaucoup de genres photographiques ne trouvent pas de localisation précise : la photo de mode par exemple, qui est très interstitielle, se situe au croisement entre statut artistique, statut documentaire et statut publicitaire.

La photographie de voyage peut être sémantisée en tant que reportage tout à fait exhaustif donc être assumée à l'intérieur du statut documentaire et en même temps avoir des ambitions artistiques comme le démontrent tous les reportages de voyage d'auteurs qui se présentent comme des journaux intimes. Mais la photographie de voyage se diffracte encore en plusieurs sous-genres : c'est le cas de la photographie touristique, qui n'est à priori pas vraiment une photographie documentaire, ni une photographie artistique, mais qui peut arriver à constituer un parcours exemplaire et assumer une fonction documentaire, ainsi que construire des points de vue sur des paysages et des villes tellement novateurs au point d'être assumée comme photographie artistique. On ne peut pas non plus oublier que la photographie touristique est elle aussi une photographie de la mémoire, étant donné

1 Bien que nous n'oubliions pas que ces différents tirages puissent ensuite donner à voir un même négatif de manières plutôt différentes : il existe des tirages du même négatif qui sont méconnaissables comme tirages du même négatif, mais qui peuvent cependant être considérés comme des exemplaires authentiques.

2 De plus, si on arrive à écrire sur les photos, comme le font beaucoup d'artistes d'aujourd'hui, cette écriture, en tant que calligraphie apposée sur la surface d'une photographie, convertit le régime d'instauration d'autographique multiple à celui d'autographique unique. 
qu'elle peut être assumée comme photo de voyage familial et rentrer par conséquent dans des pratiques de sacralisation typiques de la photo de famille. Dans ce cas, les pratiques de sacralisation de l'objet-photo sont très différentes de celles qui existent dans le domaine artistique, même si toutes deux visent la sacralisation d'un objet unique, qui dans le cas de la photo de famille est rendu pertinent par un support manipulable, tangible, valorisé pour sa biographie et son vieillissement. Au contraire, la sacralisation de l'objet-photo au statut artistique valorise un objet-photo qui ne peut pas être touché ni utilisé : il doit être gardé parfait et intact.

L'objet artistique est valorisé comme objet unique parce qu'il relève de la main et de l'œil de l'artiste qui l'a produit et qui en a décidé le type de tirage, la qualité du papier, les configurations luministiques qui sont originelles et uniques ; au contraire, dans le cas de la photographie de famille, la photo devient « unique » et sacralisée par le biais de la patine, à savoir par la trace laissée par le temps sur l'objet-photo. Dans le cas de la photo artistique, c'est la production de la photo de la part d'un certain auteur qui produit un effet de sacralisation (notamment on sacralise la corporéité de l'auteur et les configurations de la photo), dans l'autre cas, celui de la photo de famille, c'est la biographie de la photo qui en fait un objet de mémoire et de culte (ici on a affaire à un processus de sacralisation de la corporéité des usagers et de la matérialité de la photo) ${ }^{1}$.

On a un exemple célèbre de la sacralisation de la photo de famille dans La Chambre claire de Barthes, qui sacralise la photo de sa mère au Jardin d'Hiver parce que cet objet-photo a vieilli et a sauvegardé une patine qui fait remonter Barthes dans le temps jusqu'à la jeunesse de sa mère : c'est le papier couleur «sépia pâli » qui a produit cette patine sacralisante de l'objet-photo.

À partir de ces exemples on s'aperçoit que la distinction entre photo artistique et photo documentaire n'est porteuse de signification que si on multiplie les sous-distinctions par genres. Je me propose maintenant d'essayer d'enquêter sur la distinction entre photo artistique et photo scientifique, qui contribue à mieux articuler la distinction entre photo à statut artistique et photo à statut documentaire.

1 À propos des transformations matérielles des objet-photos, voir E. EDWARDS \& J. HART (sous la dir. de), Photographs, Objects, Histories. On the materiality of images, London and New York, Routledge, 2004. 


\section{Goodman entre art et science}

Si très souvent l'image artistique est signifiante pour elle-même, l'image scientifique au contraire prend sa signification de la série d'images à l'intérieur de laquelle elle est produite, outre que de sa fonction d'instrument de connaissance et de son efficacité dans la résolution de problèmes. L'image scientifique n'est jamais une image isolée. Des images scientifiques, on doit toujours pouvoir récupérer les images qui les ont précédées et/ou générées, les paramètres de production qui les ont rendues possibles et grâce auxquels elles acquièrent le statut d'image justifiée et justifiable.

En effet en partant des distinctions du philosophe Nelson Goodman entre autographie et allographie on a affirmé que la photographie artistique est un exemple d'image autographique comme c'est le cas du tableau, parce qu'elle ne possède pas de notation, comme c'est le cas dans les arts allographiques. Les textes autographiques ne sont pas engendrés par d'alphabet de signes, de grammaire, bref, ils ne peuvent pas être «exécutés » et ré-exécutés comme c'est le cas, en revanche, des textes allographiques telle la partition musicale (là on a un alphabet de signes, une notation, des exécutions dont on peut mesurer l'exactitude). La photo scientifique, contrairement à la photographie artistique, est produite par une partition, obtenue par prélèvement de données et calcul, qu'on doit littéralement exécuter (l'exemple le plus banal est celui des images produites en astrophysique et de l'imagerie médicale).

Rappelons que Nelson Goodman conçoit la notation comme un ensemble de règles de composition ; la notation assure la possibilité de multiples exécutions, comme c'est le cas de la partition musicale, parce qu'elle se manifeste comme un ensemble de signes disjoints et différenciés, donc ré-employables et re-combinables. La notation est composée de signes articulés et elle fonctionne comme un texte générateur d'exécutions. Au contraire, le régime autographique concerne les textes denses qui ne sont pas reproductibles et ré-exécutables à travers des règles. Les zones denses sont des zones où tous les traits sont pertinents à la signification.

Il existe cependant une contrôlabilité et manipulabilité de l'image scientifique qu'on ne trouve pas dans la photo artistique (en témoignent les pratiques de droits d'auteurs, et l'interdiction de la moindre modification dans la phase du tirage). La photo scientifique, à travers cette contrôlabilité, se pose de l'autre côté des pratiques de sémantisation de 
l'image artistique qui joue sur la sacralisation des originaux ; bien au contraire, les pratiques de manipulation de l'image scientifique demandent à être partagées, partageables et modifiables. C'est la manipulabilité (par filtrage, par exagération des couleurs, etc.) de l'image scientifique qui la rend efficace.

Les images artistiques sont des images qui, par excellence, ne sont pas manipulables ; elles doivent être interprétées comme des globalités, ce qui n'arrive ni dans le cas de l'image scientifique qui met en scène des résultats obtenus, ni dans celle sortie de calculs. Les images scientifiques sont là pour être transformées, essayées, vérifiées, alors que les images artistiques doivent être interprétées comme des blocs fixes. Les images artistiques sont uniques, définitives, autographiques : il n'y a pas de paramètres ni de mesures vérifiables.

\section{Pour conclure}

On voit bien que les pratiques de manipulation de l'image scientifique se situent à l'opposé des pratiques de sacralisation de l'image artistique. Entre ses deux pratiques « extrêmes » on peut situer le statut documentaire de l'image photographique, image qui est toujours lue à travers deux axes de pertinence : d'abord celui du témoignage de quelque chose qui «a été » et qui permet de déclencher des investigations (par exemple des investigations policières, politiques, etc.), et ensuite celui du témoignage de quelque chose qui « pourra être », et qui permet de se connaître mieux soi-même et sa propre perception du monde. 
